\title{
Modelling interstellar extinction and polarization with spheroidal grains
}

\author{
N.V. Voshchinnikov ${ }^{a, 1}$ H.K. Das ${ }^{b}$ \\ ${ }^{a}$ Sobolev Astronomical Institute, St. Petersburg University, St. Petersburg, 198504 Russia \\ ${ }^{\mathrm{b}}$ IUCAA,Post Bag 4, Ganeshkhind, Pune 411 007, India \\ (Received .. October 2007)
}

\begin{abstract}
We calculate the wavelength dependence of the ratio of the linear polarization degree to extinction (polarizing efficiency) $P(\lambda) / A(\lambda)$ from the ultraviolet to near-infrared. The prolate and oblate particles with aspect ratios from $a / b=1.1$ up to 10 are assumed to be rotating and partially aligned with the mechanism of paramagnetic relaxation (DavisGreenstein). Size/shape/orientation effects are analyzed. It is found that the wavelength dependence of $P(\lambda) / A(\lambda)$ is mainly determined by the particle composition and size whereas the values of $P(\lambda) / A(\lambda)$ depend on the particle shape, degree and direction of alignment.
\end{abstract}

Key words: Light scattering; Non-spherical particles; Extinction; Polarization

\section{Introduction}

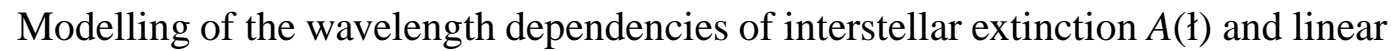
polarization $P(\nmid)$ allows one to obtain information about such properties of interstellar grains as size, composition, shape, etc. Interstellar polarization also tells us about the structure of magnetic fields because it arises due to dichroic extinction of non-spherical grains aligned in large-scale Galactic magnetic fields [1], [2]. A correlation between observed interstellar extinction and polarization shows that the same particles are responsible for both phenomena.

Very often interstellar extinction and polarization are modelled separately (e.g., [3], [4]). The modelling of these phenomena usually includes consideration of normal-

1 To whom all correspondence should be addressed. Phone: (+7) 812/428 42 63; Fax: (+7) 812/428 71 29; e-mail:nvv@ astro.spbu.ru 
ized extinction $E(\lambda-\mathrm{V}) / E(\mathrm{~B}-\mathrm{V})$ and normalized polarization given by Serkowski's curve ( $P_{\max }$ is the maximum degree of polarization and $\lambda_{\max }$ the wavelength corresponding to it, $K$ is the coefficient) $P(\lambda) / P_{\max }=\exp \left[-K \ln ^{2}\left(\lambda_{\max } / \lambda\right)\right]$ (see, e.g., [5] and discussion in [6], [7]). In these cases, the important observational data (absolute values of extinction and polarization) are ignored and normalized curves can be fitted using particles of different composition and slightly different sizes (see, e.g., discussion in [1], [7]). For example, the consideration of absolute extinction gives the possibility to use the cosmic abundances to constrain grain models (see [8]). In the case of interstellar polarization it is better to interpret the wavelength dependence of polarizing efficiency per unit extinction $P(\lambda) / A(\lambda)$ (ratio of the linear polarization degree to extinction). This allows one to estimate the degree and direction of grain alignment and particle shape. Note that previous consideration of polarizing efficiency was restricted by one wavelength band only (like $P(\mathrm{~V}) / A(\mathrm{~V})$ or $P(\mathrm{~K}) / A(\mathrm{~K}))$.

Here, we analyze the extinction and polarization produced by homogeneous spheroidal particles with different aspect ratios $a / b$ ( $a$ and $b$ are major and minor axes, respectively). Spheroidal particles have the important applications in various fields of science (spheroidal antennas, bacteria and microweeds, raining drops, etc.). By changing $a / b$ the particles with a shape varying from close to as sphere $(a / b \simeq 1)$ up to needles (prolate spheroids) or disks (oblate spheroids) can be studied. The particles are assumed to be rotating and partially aligned with the Davis-Greenstein mechanism (mechanism of paramagnetic relaxation) [9]. We focus on the behaviour of polarizing efficiencies and consider the normalized extinction $A(\lambda) / A_{\mathrm{V}}$ (i.e., ignore the cosmic abundances which will be taken into account in next paper). The theory is compared with observations of stars seen apparently through one interstellar cloud.

\section{Polarizing efficiency: observations}

Interstellar extinction grows with a radiation wavelength decrease while interstellar linear polarization has a maximum in the visual part of spectrum and declines at shorter and longer wavelengths (see [6], [7] and Fig. 1, left panel).

The polarizing efficiency of the diffuse interstellar medium is defined as the ratio of the percentage polarization $(P)$ to the extinction $(A)$ observed at the same wavelength $P(\lambda) / A(\lambda)$. There exists an empirically found upper limit on this ratio

$$
P_{\max } / A(\mathrm{~V}) \lesssim 3 \% / \mathrm{mag},
$$

where $P_{\max }$ is the maximum degree of linear polarization which is reached on average near the wavelength $\lambda_{\max } \approx 0.55 \mu \mathrm{m}$ (see [6], [7] for more discussion). 

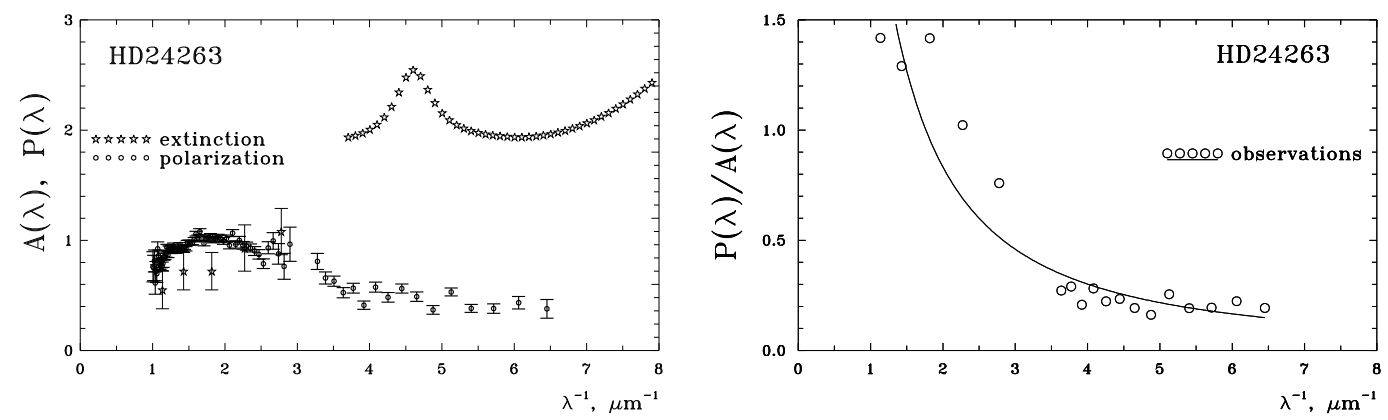

Fig. 1. Interstellar extinction (stars and thin line) and polarization curves for star HD 24263 (left panel) and the polarizing efficiency of the interstellar medium in the direction of this star (right panel; solid curve shows the power-law approximation $P / A \propto \lambda^{1.47}$ ). Observational data were taken from [10] (extinction) and [11] (polarization).

We chose five stars located not far than $500 \mathrm{pc}$ with measured ultraviolet (UV) polarization [11], then found the extinction (data published in [10] were mainly used) and calculated the ratio $P(\lambda) / A(\lambda)$. It is suggested that these stars are seen through one interstellar cloud that is supported by a weak rotation of positional angle [11]. The obtained polarizing efficiencies are shown in Fig. 2. Apparently, first presentation of the observational data in the similar form was made by Whittet ([12], Fig. 9) who gave the average normalized dependence $P(\lambda) / A(\lambda) \cdot A_{\mathrm{V}} / P_{\max }$.

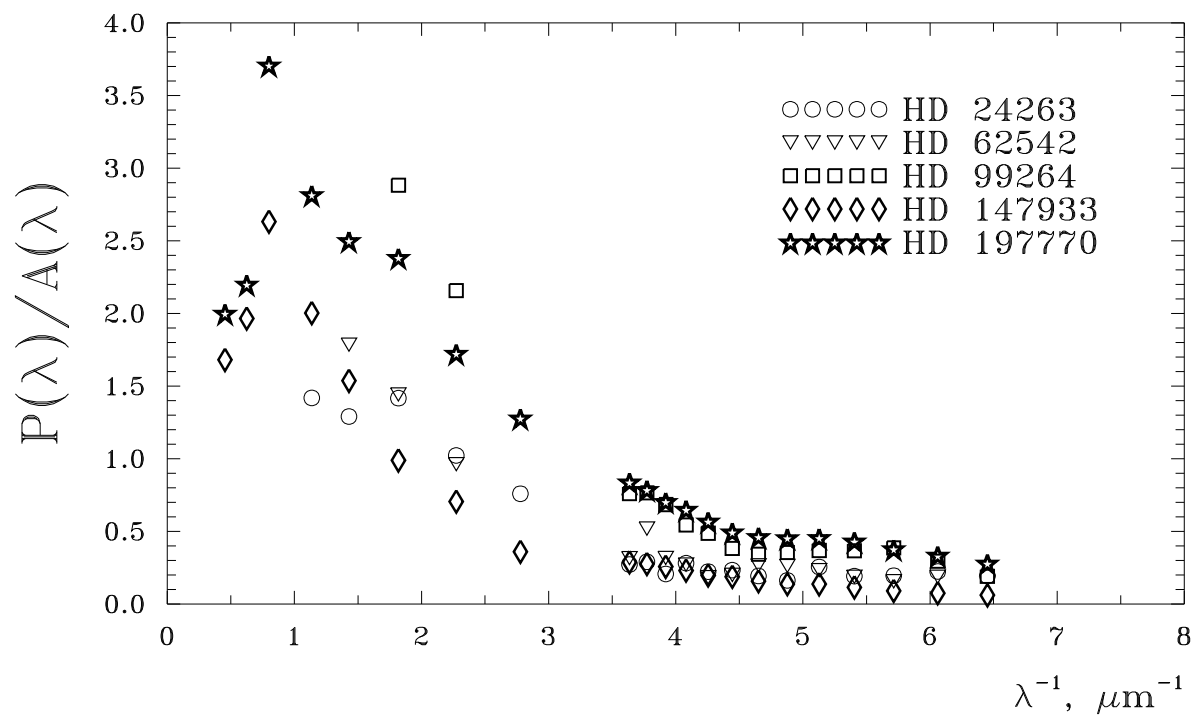

Fig. 2. Polarizing efficiency of the interstellar medium in the direction of five stars. Observational data were taken from [10] (extinction) and [11] (UV and visual polarization), [13] (IR polarization).

Note that the polarizing efficiency generally increases with a wavelength growth if $\lambda \lesssim 1 \mu \mathrm{m}$. It may be approximated using the power-law dependence $P / A \propto \lambda^{\epsilon}$. For stars presented in Fig. 2 the values of $\epsilon$ vary from 1.41 for HD 197770 to 2.06 for HD 99264. 


\section{Modelling}

Let us consider non-polarized light passing through a cloud of rotating spheroidal grains. Interstellar grains are usually partially aligned (see, e.g., [14]). Imperfect alignment is also supported by the fact that values of the polarizing efficiencies calculated for non-rotating or perfectly aligned particles are generally higher than the empirically estimated upper limit given by Eq. (1) [7], [15].

The extinction in stellar magnitudes and polarization in percentage can be found as [7]

$$
A(\lambda)=1.086 N_{\mathrm{d}}\left\langle C_{\mathrm{ext}}\right\rangle_{\lambda}, \quad P(\lambda)=N_{\mathrm{d}}\left\langle C_{\mathrm{pol}}\right\rangle_{\lambda} 100 \%,
$$

where $N_{\mathrm{d}}$ is the dust grain column density and $\left\langle C_{\mathrm{ext}}\right\rangle_{\lambda}$ and $\left\langle C_{\mathrm{pol}}\right\rangle_{\lambda}$ are the extinction and linear polarization cross sections, respectively, averaged over the grain orientations

$$
\begin{aligned}
& \left\langle C_{\mathrm{ext}}\right\rangle_{\lambda}=\left(\frac{2}{\pi}\right)^{2} \int_{0}^{\pi / 2} \int_{0}^{\pi / 2} \int_{0}^{\pi / 2} \pi r_{V}^{2} Q_{\mathrm{ext}} f(\xi, \beta) d \varphi d \omega d \beta, \\
& \left\langle C_{\mathrm{pol}}\right\rangle_{\lambda}=\frac{2}{\pi^{2}} \int_{0}^{\pi / 2} \int_{0}^{\pi} \int_{0}^{\pi / 2} \pi r_{V}^{2} Q_{\mathrm{pol}} f(\xi, \beta) \cos 2 \psi d \varphi d \omega d \beta .
\end{aligned}
$$

Here, $r_{V}$ is the radius of a sphere with the same volume as spheroidal grain, $\beta$ is the precession-cone angle for the angular momentum $\mathbf{J}$ which spins around the magnetic field direction $\mathbf{B}, \varphi$ the spin angle, $\omega$ the precession angle (see Fig. 1 in [15]). The quantities $Q_{\mathrm{ext}}=\left(Q_{\mathrm{ext}}^{\mathrm{TM}}+Q_{\mathrm{ext}}^{\mathrm{TE}}\right) / 2$ and $Q_{\mathrm{pol}}=\left(Q_{\mathrm{ext}}^{\mathrm{TM}}-Q_{\mathrm{ext}}^{\mathrm{TE}}\right) / 2$ are, respectively, the extinction and polarization efficiency factors for the non-polarized incident radiation, $f\left(\xi, r_{V}\right)$ is the cone-angle distribution.

We consider so-called imperfect Davis-Greenstein (IDG) orientation [9]. The IDG mechanism is described by the function $f(\xi, \beta)$ which depends on the alignment parameter $\xi$ and the angle $\beta$

$$
f(\xi, \beta)=\frac{\xi \sin \beta}{\left(\xi^{2} \cos ^{2} \beta+\sin ^{2} \beta\right)^{3 / 2}} .
$$

The parameter $\xi$ depends on the particle size $r_{V}$, the imaginary part of the grain magnetic susceptibility $\chi^{\prime \prime}\left(=\varkappa \omega_{\mathrm{d}} / T_{\mathrm{d}}\right.$, where $\omega_{\mathrm{d}}$ is the angular velocity of grain), gas density $n_{\mathrm{g}}$, the strength of magnetic field $B$ and dust $\left(T_{\mathrm{d}}\right)$ and gas $\left(T_{\mathrm{g}}\right)$ temper- 
atures

$$
\xi^{2}=\frac{r_{V}+\delta_{0}\left(T_{\mathrm{d}} / T_{\mathrm{g}}\right)}{r_{V}+\delta_{0}}, \quad \text { where } \quad \delta_{0}^{\mathrm{IDG}}=8.2310^{23} \frac{\varkappa B^{2}}{n_{\mathrm{g}} T_{\mathrm{g}}^{1 / 2} T_{\mathrm{d}}} \mu \mathrm{m} .
$$

The angle $\psi$ in Eq. (4) is expressed via the angles $\varphi, \omega, \beta$ and $\Omega$ (angle between the line of sight and the magnetic field, $0^{\circ} \leq \Omega \leq 90^{\circ}$ ). Note that for the case of the perfect DG orientation (PDG, perfect rotational or 2D orientation) the major axis of a non-spherical particle always lies in the same plane. For PDG, integration in Eqs. (3), (4) is performed over the spin angle $\varphi$ only.

As usual, the particles of different sizes are considered. In this case the crosssections $\left\langle C_{\text {ext }}\right\rangle_{\lambda}$ and $\left\langle C_{\text {pol }}\right\rangle_{\lambda}$ are obtained after the averaging over size distribution function $n\left(r_{V}\right)$

$$
\int_{r_{V, \min }}^{r_{V, \min }}\langle C\rangle_{\lambda}\left(r_{V}\right) n\left(r_{V}\right) d r_{V}
$$

where $r_{V, \min }$ and $r_{V, \max }$ are the lower and upper cutoffs, respectively.

We choose the power-law size distribution function

$$
n\left(r_{V}\right) \propto r_{V}^{-q}
$$

which was often used for the modelling of interstellar extinction [16], [17]. The average Galactic extinction curve can be reproduced using the mixture of carbonaceous (graphite) and silicate particles (in almost equal proportions) with parameters: $q=3.5, r_{V, \min } \approx 0.001 \mu \mathrm{m}$ and $r_{V, \max } \approx 0.25 \mu \mathrm{m}$.

\section{Results and discussion}

Our calculations have been performed for prolate and oblate rotating spheroids of several sizes and aspect ratios. The particles consisting of astronomical silicate (astrosil) and amorphous carbon (AC1) were considered. The optical properties of spheroids were derived using the solution to the light scattering problem by the separation of variables method [18] and modern treatment of spheroidal wave functions [19].

Below we briefly discuss extinction by spheroids and in sufficient detail polarizing efficiency. The comparison with observations of two stars is performed in Sect. 4.3 . 


\subsection{Extinction curve}

Extinction (and sometimes polarization) produced by spheroids was studied by Rogers and Martin [20], Onaka [21], Vaidya et al. [22], Voshchinnikov [23] and more recently by Gupta et al. [24]. Mostly the particles with the aspect ratio $a / b \leq 2$ were considered.

The parameters of our model (see Sect 3) are: the type (prolate or oblate) and composition of spheroids, the size $r_{V}$ or the parameters of the size distribution $r_{V \text {,min }}$, $r_{V \text {,max }}, q$, the degree specified through parameter $\delta_{0}$ and direction of alignment $\Omega$. Figure 3 illustrates how variations of particle shape and alignment influence on the normalized extinction $A(\lambda) / A_{V}$. The value of $\delta_{0}$ for IDG orientation is typical of diffuse interstellar medium [15]. It is seen that the difference in curves are in evidence only in the UV. Prolate spheroids produce larger extinction when the angle of alignment reduces from $\Omega=90^{\circ}$ (magnetic field is perpendicular to the line of site) to $\Omega=0^{\circ}$ (magnetic field is parallel to the line of site; Fig. 3, left panel). For oblate particles the dependence on $\Omega$ is opposite. However, only for PDG these changes are at a detectable rate. Extinction also grows when the particles (prolate and oblate) become more elongated or flattened (see Fig. 3, right panel).
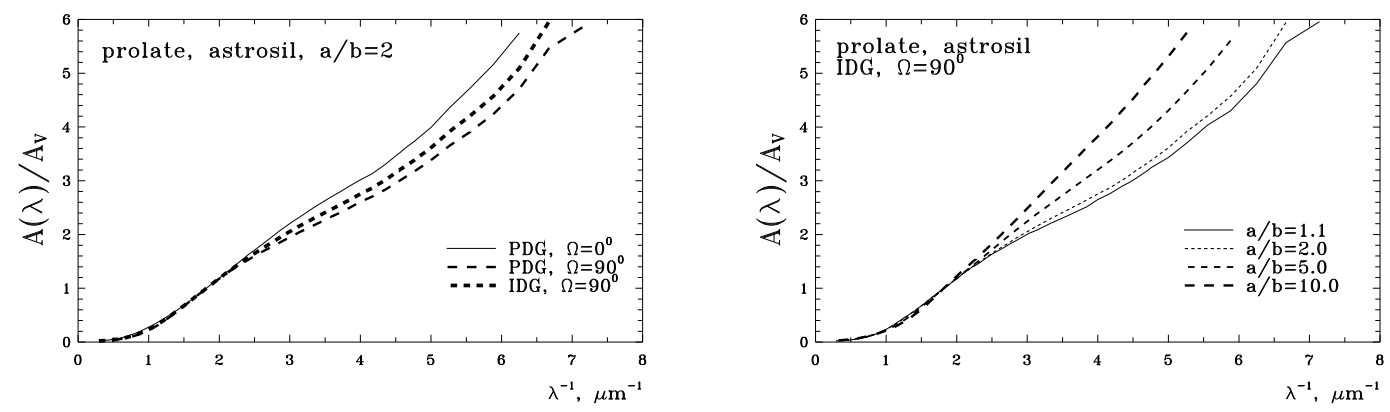

Fig. 3. Normalized extinction for ensembles of aligned prolate spheroids from astrosil. Spheroids have power-law size distribution with parameters: $r_{V, \min }=0.001 \mu \mathrm{m}$, $r_{V, \max }=0.25 \mu \mathrm{m}$ and $q=3.5 ; \delta_{0}=0.196 \mu \mathrm{m}$. The effect of variations of particle degree and direction of alignment (left panel) and particle shape (right panel) is illustrated.

Variations of parameters of size distribution $\left(r_{V, \min }, r_{V, \max }, q\right)$ on extinction are similar to those for spheres are not discussed here (see, e.g., [25]).

\subsection{Polarizing efficiency: theory}

Theoretical dependence of the polarizing efficiency of the interstellar medium was discussed several times for particles of constant refractive index (see, e.g., [20], [21]). The wavelength dependence of $P / A$ was considered in [26] for infinite and finite cylinders and in [7] for perfectly aligned spheroids of single size. Here, we 
analyse firstly the dependence $P(\lambda) / A(\lambda)$ for single size particles and then for ensembles with a size distribution.

\subsubsection{Single size}

Some results for prolate particles are plotted in Figs. 4 and 5 . They show the polarizing efficiency in the wavelength range from the infrared to far ultraviolet. The dependence $P(\lambda) / A(\lambda)$ observed for two stars is given for comparison. We made calculations for compact grains and for porous grains using the Bruggeman mixing rule for refractive index and particles of same mass as compact ones. Note that calculated efficiencies can be considered as upper limits because some populations of grains (spherical, non-oriented) may contribute into extinction but not to polarization.
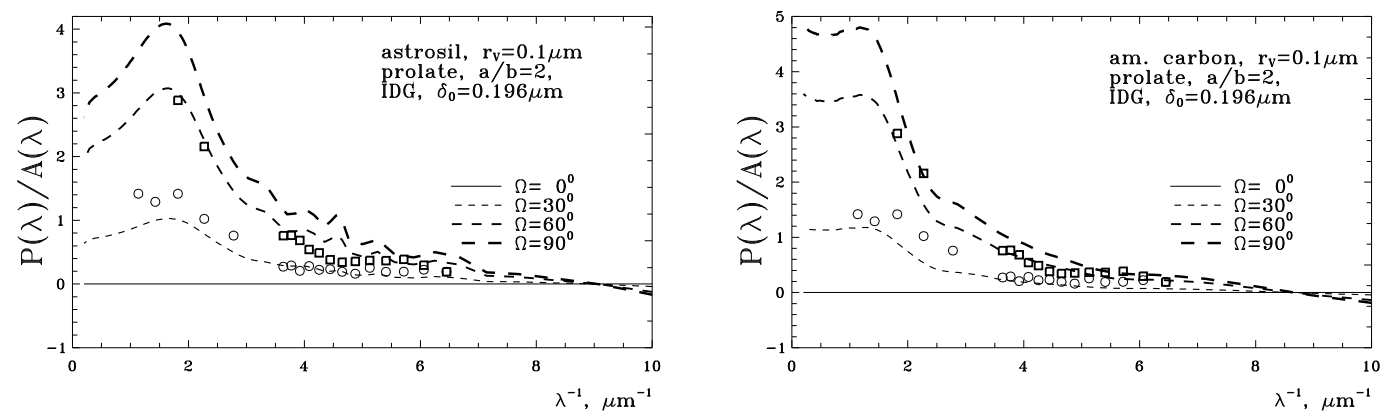

Fig. 4. Wavelength dependence of polarizing efficiency for homogeneous rotating spheroidal particles of astronomical silicate and amorphous carbon. The effect of variations of particle composition and direction of alignment is illustrated. The open circles and squares show the observational data for stars HD 24263 and HD 99264, respectively.

From Figs. 4, 5] one can conclude that the wavelength dependence of polarizing efficiency is mainly determined by the particle composition and size. Variations of other parameters influence on the value of efficiency (the dependence of $P / A$ is scaled). A growth of the efficiencies $P(\lambda) / A(\lambda)$ takes the place if we increase angle $\Omega$ (direction of alignment deviates widely from the line of site), parameter $\delta_{0}$ (degree of alignment) or aspect ratio $a / b$ (consider more elongated or flattened particles) and decrease particle porosity (volume fraction of vacuum) $\mathcal{P}$ or particle size $r_{V}$. It is also evident that the contribution of particles with size $r_{V} \approx 0.1 \mu \mathrm{m}$ to observed polarization must be rather important and the polarization produced by perfectly aligned particles significantly exceeds the observed one.

\subsubsection{Size distribution}

We also calculated the wavelength dependencies of $P / A$ separately for spheroids from $\mathrm{AC} 1$ and astrosil with standard power-law size distribution $\left(r_{V, \min }=0.001 \mu \mathrm{m}\right.$, $r_{V, \max }=0.25 \mu \mathrm{m}$ and $\left.q=3.5\right)$ and considered the effect of variations different model parameters. The results are shown in Figs. 6, and 7 Note again that the value of $P / A$ 

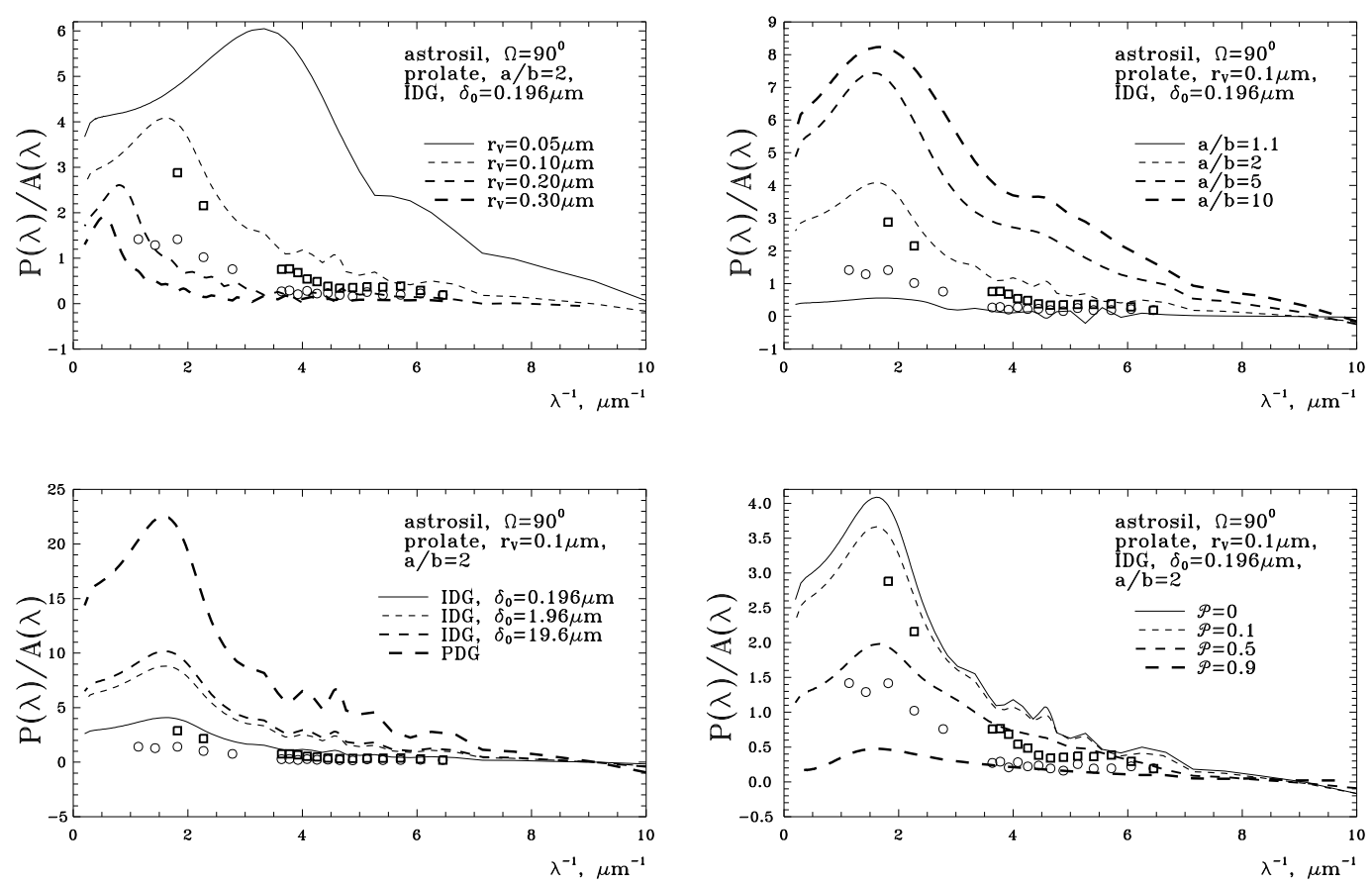

Fig. 5. Wavelength dependence of polarizing efficiency for homogeneous rotating spheroidal particles of astronomical silicate. The effect of variations of particle size, shape, porosity and degree of alignment is illustrated. The open circles and squares show the observational data for stars HD 24263 and HD 99264, respectively.

depends on the degree and direction of alignment and the shape of particles while the particle composition and size strongly influences on the wavelength dependence of polarizing efficiency.
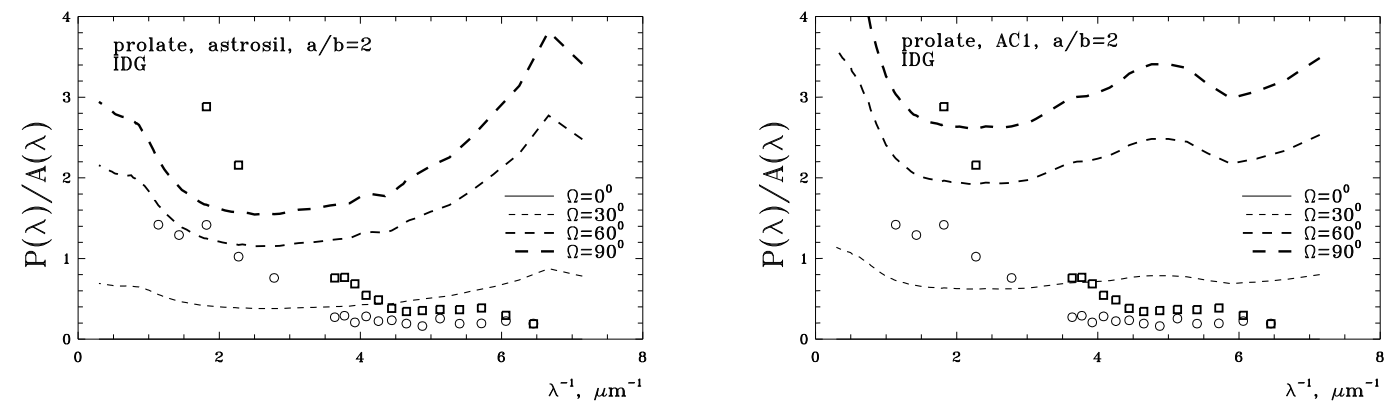

Fig. 6. Polarizing efficiency ensembles of aligned prolate spheroids with $a / b=2$ from astrosil (left panel) and amorphous carbon (right panel). Spheroids have power-law size distribution with parameters: $r_{V, \min }=0.001 \mu \mathrm{m}, r_{V, \max }=0.25 \mu \mathrm{m}$ and $q=3.5 ; \delta_{0}=0.196 \mu \mathrm{m}$. The effect of variations of particle composition and direction of alignment is illustrated. The open circles and squares show the observational data for stars HD 24263 and HD 99264, respectively.

As follows from these Figures, theoretical polarizing efficiency more or less resembles the observational dependencies only if the contribution of small particles into polarization is depressed $\left(r_{V, \min }>0.05 \mu \mathrm{m}\right.$ or $q<3$, see Fig. 7). It is well known for a long time that the polarization of forward transmitted radiation is determined by 

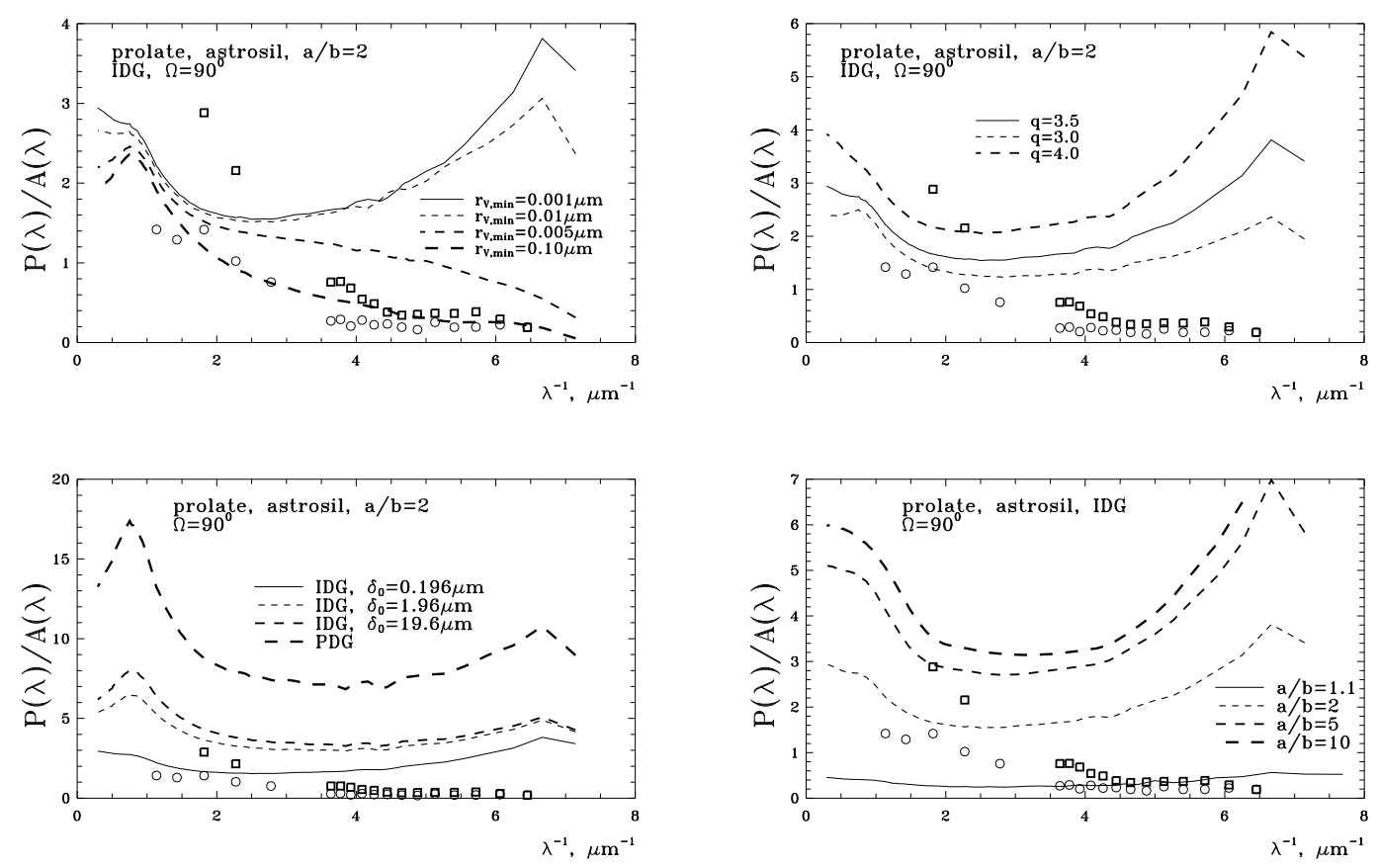

Fig. 7. Polarizing efficiency ensembles of aligned prolate spheroids from astrosil. Spheroids have power-law size distribution, $\Omega=90^{\circ}$. The effect of variations of parameters of size distribution, degree of alignment and particle shape is illustrated. The open circles and squares show the observational data for stars HD 24263 and HD 99264, respectively.

particles of small sizes while large particles produce no polarization independent of their shape (see discussion in [1] and [27]).

The influence of small particles on $P / A$ is reduced if: a) the particles are absent in the interstellar cloud, b) the small particles are less oriented or c) the shape of small particles is closer to spherical. The reason a) must manifests itself by the depressed UV extinction while the reasons b) and c) would be difficult to distinguish.

The relationships found can be used for more detailed comparison of the theory with observations.

\subsection{Comparison with observations}

We calculated the normalized extinction $A(\lambda) / A_{V}$ and the polarizing efficiency and compared the results with observations of stars mentioned in Sect. 2. In order to fit the UV bump on the extinction curve a small amount of graphite spheres with radius $r_{V} \approx 0.015 \mu \mathrm{m}$ was added to the mixture of silicate and carbon spheroids.

According to the model presented in Sect. 3, we adopted that the same particles produce extinction and polarization and the alignment of silicate and carbon particles is identical. At the same time, parameters of size distribution for silicate and 
carbon can differ.

The results of comparison are shown for two stars in Figs. 8 and 9 .
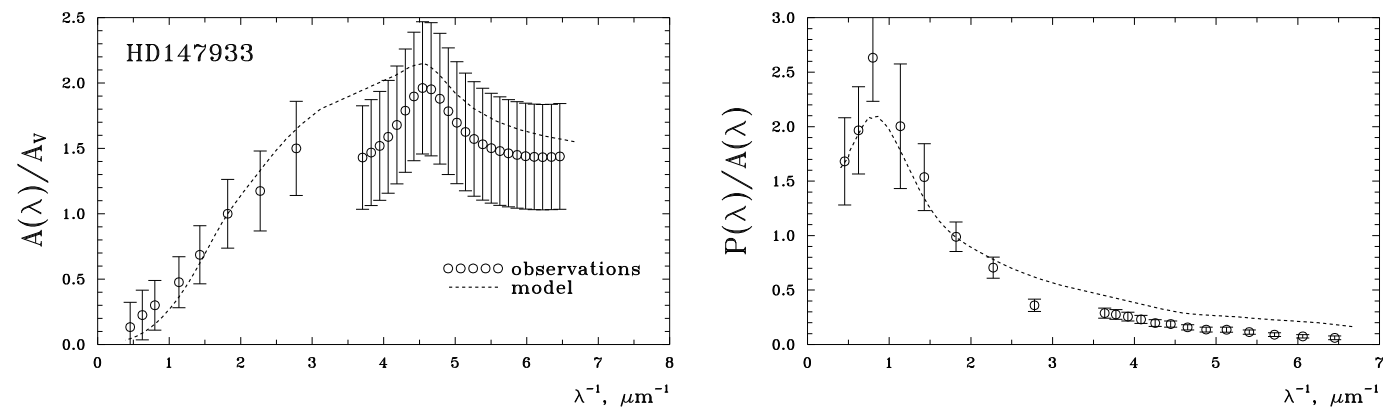

Fig. 8. Normalized extinction (left panel) and the polarizing efficiency (right panel) in the direction of the star HD 147933. The error bars show $1 \sigma$ uncertainty. Dashed curves show the results of calculations for model with prolate spheroids with $a / b=5$ from amorphous carbon (24\%) and astrosil (73\%) and graphite spheres (3\%). Spheroids have a power-law size distribution with the parameters: amorphous carbon, $r_{V, \min }=0.03 \mu \mathrm{m}$, $r_{V, \max }=0.15 \mu \mathrm{m}, q=1.5$; astrosil, $r_{V, \min }=0.08 \mu \mathrm{m}, r_{V, \max }=0.25 \mu \mathrm{m}, q=1.5$. Alignment parameters are: $\delta_{0}=0.5 \mu \mathrm{m}, \Omega=35^{\circ}$.

The coincidence of the theory with observations is rather good. The better agreement can be achieved if we assume that the alignment parameters for silicate and carbonaceous grains differ. For example, it is possible to consider non-aligned carbonaceous grains (as it was made in [28]). Evidently, more attention should be given to modern alignment mechanisms.
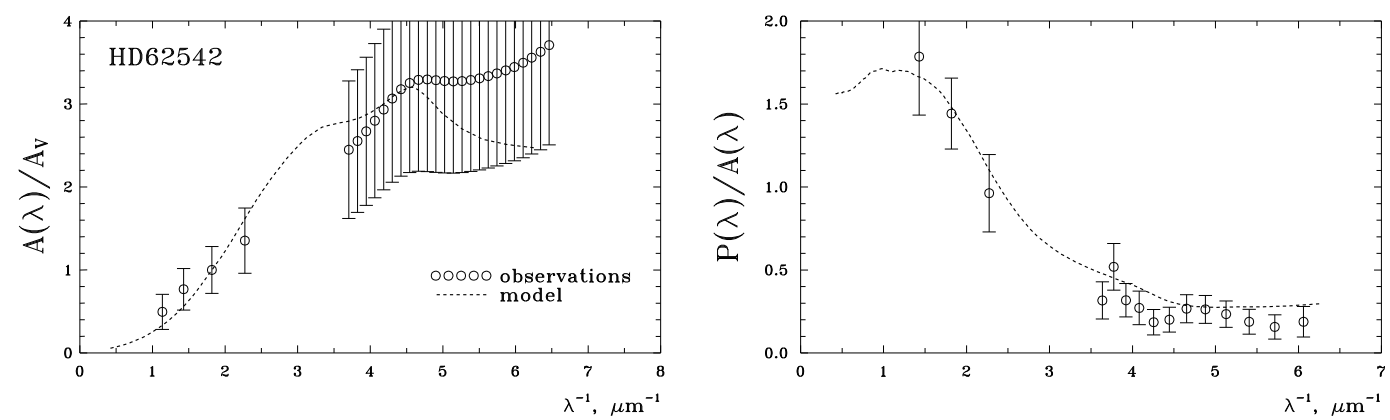

Fig. 9. Normalized extinction (left panel) and the polarizing efficiency (right panel) in the direction of the star HD 62542. The error bars show $1 \sigma$ uncertainty. Dashed curves show the results of calculations for model with prolate spheroids with $a / b=2$ from amorphous carbon $(85 \%)$ and astrosil $(10 \%)$ and graphite spheres $(5 \%)$. Spheroids have a power-law size distribution with the parameters: amorphous carbon, $r_{V, \min }=0.07 \mu \mathrm{m}, r_{V, \max }=0.10 \mu \mathrm{m}$, $q=2.7$; astrosil, $r_{V, \text { min }}=0.005 \mu \mathrm{m}, r_{V, \max }=0.25 \mu \mathrm{m}, q=2.7$. Alignment parameters are: $\delta_{0}=0.5 \mu \mathrm{m}, \Omega=40^{\circ}$.

These improvements of the model require to invoke additional observational data such as dust-phase abundances in the directions of considered stars, information about magnetic fields, etc. Otherwise, the modelling cannot give non-ambiguous results. In particular, the important feature is the wavelength where the ratio $P / A$ 
reaches a maximum (see Figs. 447). In order to find this quantity we need the observations of polarization in the near-IR (J, H, K bands) which are absent for some considered stars (Fig. 2).

\section{Conclusions}

In the frame of the model of partially aligned spheroidal grains we studied the wavelength dependence of the ratio of the linear polarization degree to extinction (polarizing efficiency) $P(\lambda) / A(\lambda)$.

The main results can be summarized as follows.

1. It is found that the wavelength dependence of $P(\lambda) / A(\lambda)$ is mainly determined by the particle composition and size.

2. The values of $P(\lambda) / A(\lambda)$ depend on the particle shape, degree and direction of alignment.

3. The modelling of the only wavelength dependence of polarizing efficiency does not allow one to determine all parameters dust ensemble. Therefore, interpretation of the observations must include consideration of extinction.

\section{Acknowledgements}

We are thankful to Vladimir Il'in for careful reading of manuscript. The work was partly supported by grants NSh 8542.2006.2, RNP 2.1.1.2152 and RFBR 07-0200831 of the Russian Federation.

\section{References}

[1] Greenberg JM. Interstellar Grains. In: Middlehurst BM, Aller LH, editors. Stars and Stellar Systems, Vol. VII, Chicago: Univ. Chicago Press, 1968, p. 221-364.

[2] Hough JH, Aitken DK. Infrared polarimetry of interstellar dust, In: Videen G et al. editors. Photopolarimetry in remote sensing Kluwer, 2004, p. 325-349.

[3] Weingartner JC, Draine BT. Dust grain-size distribution and extinction in the Milky Way, Large Magellanic Cloud, and Small Magellanic Cloud. Astrophys J 2001;548:296-309.

[4] Kim S-H, Martin PG. The size distribution of interstellar dust particles as determined from polarization: spheroids. Astrophys J 1995;444:293-305. 
[5] Wolff MJ, Clayton GC, Meade MR. Ultraviolet interstellar linear polarization I. Applicability of current grain models. Astrophys J 1993;403:722-735.

[6] Whittet DCB. Dust in the Galactic Environments. 2nd ed., Bristol: Institute of Physics Publishing, 2003.

[7] Voshchinnikov NV. Optics of Cosmic Dust. I. Astrophys Space Phys Rev 2004;12:1182.

[8] Voshchinnikov NV, Il'in VB, Henning Th, Dubkova DN. Dust extinction and absorption: the challenge of porous grains. Astron Astrophys 2006;445:167-177.

[9] Davis L, Greenstein JL. The polarization of starlight by aligned dust grains. Astrophys J 1951;114:206-239.

[10] Valencic LA, Clayton GC, Gordon KD. Ultraviolet extinction properties in the Milky Way. Astrophys J 2004;616:912-924.

[11] Andersen CM et al., Ultraviolet interstellar linear polarization of galactic starlight I. Observations by the Wisconsin Ultraviolet Photo Polarimeter Experiment. Astronom J 1996;112:2726-2743.

[12] Whittet DCB. Continuum polarization of starlight. In: Polarimetry of the Interstellar Medium, ASP Conf Ser, 1996;97:125-142.

[13] Wilking BA et al. The wavelength dependence of interstellar linear polarization. Astrophys J 1980;235:905-910.

[14] Lazarian A. Tracing magnetic fields with grain alignment. J Quant Spectrosc Radiat Transfer 2007;106:225-256.

[15] Voshchinnikov NV. Determination of dust properties and magnetic fields from polarimetric and photometric observations of stars. Astronom Nachrichten 1989;310:265-271.

[16] Mathis JS, Rumpl W, Nordsieck KH. The size distribution of interstellar grains. Astrophys J 1977;217:425-433.

[17] Draine BT, Lee HM, Optical properties of interstellar graphite and silicate grains. Astrophys J 1984;285:89-108.

[18] Voshchinnikov NV, Farafonov VG. Optical properties of spheroidal particles. Astrophys Space Sci 1993;204:19-86.

[19] Voshchinnikov NV, Farafonov VG. Computation of radial prolate spheroidal wave functions using Jáffe's series expansions. J Comp Math Math Phys 2003;43:1299_ 1309.

[20] Rogers C, Martin PG. On the shape of interstellar grains. Astrophys J 1979;228:450464.

[21] Onaka T. Light scattering by spheroidal grains. Ann Tokyo Astron Obs 1980;18:1-54.

[22] Vaidya DB, Bhatt HC, Desai JN. Interstellar extinction and polarization by spheroidal dust grains. Astrophys Space Sci 1984;104:323-336. 
[23] Voshchinnikov NV. Optical properties of the spheroidal dust grains. Forward transmitted radiation. Sov Astron 1990;34:535-541.

[24] Gupta R, Mukai T, Vaidya DB, Sen AK, Okada Y. Interstellar extinction by spheroidal dust grains. Astron Astrophys 2005;441:555-561.

[25] Voshchinnikov NV, Il'in VB. Interstellar extinction curve in the far and extreme ultraviolet. Sov Astron 1993;37:21-25.

[26] Li A, Greenberg JM, A unified model of interstellar dust. Astron Astrophys 1997;323:566-584.

[27] Voshchinnikov NV, Il'in VB, Henning Th, Michel B, Farafonov VG. Extinction and Polarization of Radiation by Absorbing Spheroids: Shape/Size Effects and Some Benchmarks. J Quant Spectrosc Radiat Transfer 2000;65:877-893.

[28] Mathis JS. The size distribution of interstellar grains. II. Polarization. Astrophys J 1979;232:747-753. 